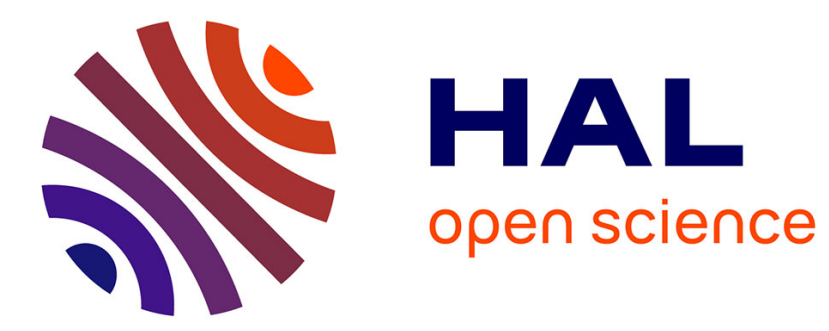

\title{
Exponents appearing in the zero-temperature dynamics of the 1D Potts model
}

\author{
Bernard Derrida
}

\section{To cite this version:}

Bernard Derrida. Exponents appearing in the zero-temperature dynamics of the 1D Potts model. Journal of Physics A: Mathematical and General, 1995, 28 (6), pp.1481-1491. 10.1088/03054470/28/6/006 . hal-03282967

\section{HAL Id: hal-03282967 https://hal.science/hal-03282967}

Submitted on 19 Jul 2021

HAL is a multi-disciplinary open access archive for the deposit and dissemination of scientific research documents, whether they are published or not. The documents may come from teaching and research institutions in France or abroad, or from public or private research centers.
L'archive ouverte pluridisciplinaire HAL, est destinée au dépôt et à la diffusion de documents scientifiques de niveau recherche, publiés ou non, émanant des établissements d'enseignement et de recherche français ou étrangers, des laboratoires publics ou privés. 


\title{
Exponents appearing in the zero-temperature dynamics of the 1D Potts model
}

\author{
B Derrida \\ Laboratoire de Physique Statistique, ENS, 24 rue Lhomond, 75005 Paris, France \\ and \\ Service de Physique Theorique, CE Saciay, F91191 Gif sur Yvette, France
}

Received 2 December 1994, in final form 16 January 1995

\begin{abstract}
For the Glauber dynamics of the one-dimensional $q$-state Potts model at zero temperature, the fraction of spins which have never fipped up to time $t$ decreases as $t^{-\theta(q)}$ where the exponent $\theta(q)$ varies continuously with $q$. The calculation of the $q$ dependence of this exponent can be reduced to the study of a simple reaction-diffusion model. Using finite-size scaling, this allows one to obtain accurate estimates of $\theta(q)$ for all values of $q$. One can also show that for $q=1+\epsilon$, the expansion of $\theta(q)$ is given by $\theta(q)=(2 / \pi) \epsilon \div O\left(\epsilon^{2}\right)$.
\end{abstract}

\section{Introduction}

The zero-temperature dynamics of one-dimensional classical spin models such as the Ising or $q$-state Potts model are known as well understood problems [1-5]. If a random initial spin configuration is quenched at zero temperature, one observes a coarsening phenomenon, with a characteristic domain size growing with $t^{1 / 2}$. It was shown [6], however, that, even for such simple systems, some dynamical properties are more difficult to understand. For example, the fraction $r(q, t)$ of spin which has never flipped [6] up to time $t$ decays with a power law

$$
r(q, t) \simeq t^{-\theta(q)}
$$

where the exponent $\theta(q)$ varies with $q$. Monte Carlo simulations [6-8] give $\theta(2) \simeq 0.376$, $\theta(3) \simeq 0.53 \theta(5) \simeq 0.70, \theta(10) \simeq 0.82$ and so far, $q=\infty$ is the only case [6] for which the exponent is known exactly $(\theta(\infty)=1)$.

In the present work, the problem of calculating the fraction $r(q, t)$ of spins which have never flipped for all values of $q$ is reduced to a single (and simple) reaction-diffusion problem: particles diffuse and aggregate $(A+A \rightarrow A)$ on a one-dimensional chain with a source of particles at the origin. If the reaction-diffusion problem starts at $t=0$ with no particle in the system (except at the origin) and if one calls $P(m, t)$, the probability that at time $t$ there are exactly $m$ particles in the system (including the one at the origin which is always present), we shall see that $r(q, t)$ is given by

$$
r(q, t)=\sum_{m=1}^{\infty} P(m, t) q^{1-m} .
$$

This relationship with the reaction-diffusion problem will allow us:

(i) to obtain the estimates in table 1 for the exponent $\theta(q)$ for integer and non-integer values of $q$; 
Table 1. Numerical values of $\theta(q)$ obtained in section 4 using finite-size scaling.

\begin{tabular}{llllllllll}
\hline$q$ & 0.5 & 1.01 & 1.5 & 2 & 3 & 5 & 10 & 100 & $\infty$ \\
\hline$\theta(q)$ & -0.513 & 0.0063203 & 0.2350 & 0.3750 & 0.5379 & 0.6928 & 0.8310 & 0.9815 & 1.0000 \\
Error & 0.001 & 0.0000002 & 0.0001 & 0.0001 & 0.0002 & 0.0003 & 0.0005 & 0.0010 & 0.0005 \\
Monte Carlo & & & & 0.376 & 0.53 & 0.70 & 0.82 & & 1.0 \\
Exact & & & & & & & & & 1 \\
\hline
\end{tabular}

(ii) to find the first term of the expansion of $\theta(q)$ in powers of $q-1$

$$
\theta(q)=\frac{2}{\pi}(q-1)+\mathrm{O}\left((q-1)^{2}\right)
$$

(iii) to obtain bounds on the exponent $\theta(q)$ : for $q_{1}<q_{2}$

$$
\theta\left(q_{2}\right) \leqslant \theta\left(q_{1}\right) \frac{\log q_{2}}{\log q_{1}}
$$

which combined with (3) gives, for $q>1$,

$$
\theta(q) \leqslant \frac{2}{\pi} \log q
$$

The paper is organized as follows. In section 2, the problem of finding the fraction $r(q, t)$ of spins which have never flipped is reduced to a simple reaction-diffusion model and $(2)$ is derived. In section 3 , the steady-state properties of the reaction-diffusion model for finite system sizes are presented. In section 4 , these results are analysed, assuming finitesize scaling to obtain accurate predictions (of table 1) for the exponent $\theta(q)$ for arbitrary values of $q$. Analytic expressions for some of the properties of the reaction-diffusion model can be obtained for arbitrary system sizes and this will be the way, in section 5 , of deriving the first term in the expansion of $\theta(q)$. Finally the bounds (4), (5) are obtained using (2) and Jensen's inequality.

\section{Relation to a reaction-diffusion model}

One can impiement the Glauber dynamics of a one-dimensional $q$-state Potts model at zero temperature as follows: during any infinitesimal time interval d $t$, each spin $S_{i}(t)$ has a probability $\mathrm{d} t / 2$ of becoming equal to its left neighbour, $\mathrm{d} t / 2$ of becoming equal to its right neighbour, and a probability $1-\mathrm{d} t$ of keeping its own value. The only difference between the different values of $q$ is in the random initial condition: at $t=0$, each $\operatorname{spin} S_{i}(0)$ is assigned a random integer between $i$ and $q$.

There is a very direct way $[2,4,5,8-11]$ of relating this coarsening phenomenon to reaction-diffusion models [12-22]: one considers that the domain walls perform random walks and when two of these random walkers meet, they either annihilate $(A+A \rightarrow \emptyset)$ with probability $1 /(q-1)$ or they aggregate $(A+A \rightarrow A)$ with probability $(q-2) /(q-1)$. These rates are easy to understand by considering what happens when two domain walls meet, i.e. when a domain disappears: either, the two neighbours of the domain which disappears are in the same state and the two domain walls annihilate; or they are in different states and the two domain walls merge into a single domain wall. This reaction-diffusion model with the fact that in the initial condition, each bond is occupied by a domain wall with probability $(q-1) / q$ is completely equivalent to the initial spin problem. Then the density $r(q, t)$ of spins which have never flipped up to time $t$ is just the fraction of sites which have never 
been jumped over by a domain wall $[6,8,9]$. This domain wall description is a useful one, but the model changes when $q$ varies and difficulties arise if one tries to consider non-integer values of $q$ (for example for $q$ close to 1 , one has to work with negative probabilities).

In this paper, a different reasoning is used to relate the calculation of the fraction $r(q, t)$ to a reaction-diffusion model. It is useful to think of the dynamics of the spin model in terms of random walks. We have seen that the value of $S_{i}(t)$ at time $t$ is equal to $S_{i-1}(t-\mathrm{d} t)$, $S_{i}(t-\mathrm{d} t)$ or $S_{i+1}(t-\mathrm{d} t)$ with probabilities $\mathrm{d} t / 2,1-\mathrm{d} t$ and $\mathrm{d} t / 2$. These spin values, in turn, depend on $S_{i-2}(t-2 \mathrm{~d} t), S_{i-1}(t-2 \mathrm{~d} t), S_{i}(t-2 \mathrm{~d} t), S_{i+1}(t-2 \mathrm{~d} t), S_{i+2}(t-2 \mathrm{~d} t)$ and so on. Tracing back in time the sequence of spins responsible for the value of $S_{i}(t)$ defines a random walk. Thus the value of spin $S_{i}(t)$ at time $t$ is equal to the value of another spin $S_{j}(0)$ at time 0 with a probability $p(j-i, t)$ where $p(j-i, t)$ is the probability for a random walk to go from $i$ to $j$ during time $t$, given that during each time interval $\mathrm{d} t$, the random walk moves to its left with probability $\mathrm{d} t / 2$, to its right with probability $\mathrm{d} t / 2$ or does not move with probability $1-\mathrm{d} t$. So to know the value of $S_{0}(t)$, one just needs to look at the position at time $t$ of a random walk starting at $t=0$ at the origin.

Assume that one wants to know the values of $S_{0}(t)$ and $S_{0}\left(t^{\prime}\right)$ with $t>t^{\prime}$. Then one has to consider the positions at time $t$ of two random walkers, the first one starting at the origin at time $t=0$ and the second one starting at the origin at time $t-t^{\prime}$. Clearly, if the two walkers occupy the same site at a given time, they follow, at any later time, the same trajectory. So the two walkers are like two diffusing particles which aggregate when they meet.

Let us call $p\left(1 ; t, t^{\prime}\right)$ the probability that the two walkers aggregate before time $t$ and $p\left(2 ; t, t^{\prime}\right)$ the probability that they do not. Then the probability that $S_{0}(t)=S_{0}\left(t^{\prime}\right)$ is given by $p\left(1, t ; t^{\prime}\right)+p\left(2 ; t, t^{\prime}\right) / q$. This reasoning can easily be extended to obtain the probability that $S_{0}(t)=S_{0}\left(t^{\prime}\right)=S_{0}\left(t^{\prime \prime}\right)=\cdots$. It follows that, to calculate the probability $r(q, t)$ that the spin at the origin has never flipped up to time $t$, one needs to consider the following reaction-diffusion problem: the system consists of random walkers which diffuse and aggregate; moreover the origin is always occupied (i.e. whenever the walker which is at the origin jumps to one of its neighbours, a new walker is produced at the origin). If initially the system is empty (except for the origin) and if one calls $P(m, t)$ the probability of having $m$ walkers in the system at time $t$, the same argument as above (when comparing $S_{0}(t)$ and $S_{0}\left(t^{\prime}\right)$ ) leads to

$$
r(q, t)=\sum_{m=1}^{\infty} P(m, t) q^{\mathrm{I}-m} .
$$

This is the origin of the equation (2) which is the starting point of the present work. Reaction-diffusion models with sources have already been studied in the literature [2,11$13,15]$. A number of results are known, but, to my knowledge, the exact expression for $P(m, t)$ has not yet been given.

\section{Steady-state properties of a finite system}

Let us consider the reaction-diffusion problem on a ring of $L$ sites. One site (which we call the origin) is always occupied and all the other sites are either occupied by one particle or empty. The particles diffuse and when two particles meet, they aggregate $(A+A \rightarrow A)$. The effect of the aggregation is to reduce the number of particles in the system whereas the source at the origin increases this number. The result is a steady state with a probablity $\psi(m, L)$ of finding $m$ sites occupied on a ring of $L$ sites. 
Clearly, a ring of $L$ sites has $2^{L-1}$ possible configurations (each site is either occupied or empty except for the origin which is always occupied). If $W\left(\mathcal{C}^{\prime}, \mathcal{C}\right) \mathrm{d} t$ is the probability during time $\mathrm{d} t$ to go from a configuration $\mathcal{C}$ to a configuration $\mathcal{C}^{\prime}$, the weights $p(\mathcal{C})$ in the steady state are solutions of

$$
\sum_{\mathcal{C}^{\prime}} W\left(\mathcal{C}^{\prime}, \mathcal{C}\right) p(\mathcal{C})=\sum_{\mathcal{C}^{\prime}} W\left(\mathcal{C}, \mathcal{C}^{\prime}\right) p\left(\mathcal{C}^{\prime}\right)
$$

For $L$ not too large, the matrix $W\left(C^{\prime}, \mathcal{C}\right)$ can be constructed explicitly by enumerating all possible configurations and all possible moves and it is then easy to find numerically the weights $p(C)$ by solving equations (7). For example for $L=4$, and if the origin (i.e. the source of particles) is at position 4, one finds the following weights in the steady state for the $2^{3}$ possible configurations

$$
\begin{array}{lll}
p(0001)=\frac{8}{44} & p(1001)=p(0011)=\frac{8}{44} & p(0101)=\frac{7}{44} \\
p(1011)=\frac{6}{44} & p(1101)=p(0111)=\frac{3}{44} & p(1111)=\frac{1}{44}
\end{array}
$$

and therefore

$$
\psi(1,4)=\frac{8}{44} \quad \psi(2,4)=\frac{23}{44} \quad \psi(3,4)=\frac{12}{44} \quad \psi(4,4)=\frac{1}{44} .
$$

Table 2 gives all the $\psi(m, L)$ for $L \leqslant 14$.

One finds that the $\psi(m, L)$ are rational numbers which are listed in table 2 (for each $m$, the numerators of $\psi(m, L)$ are given in table 2 and at the end of each list, the sum of all the numerators gives the denominator).

Remark I. So far, there are two properties of the $\psi(m, L)$ which could be obtained analytically for all $L$ : first, the exact expression for $\psi(1, L)$ is

$$
\psi(1, L)=\frac{16}{(L+1)^{2}} \sum_{k \text { even }} \sum_{k^{\prime} \text { odd }} \frac{\sin ^{2} k \alpha \sin ^{2} k^{\prime} \alpha}{\left(\cos k^{\prime} \alpha-\cos k \alpha\right)\left(2-\cos k \alpha-\cos k^{\prime} \alpha\right)}
$$

second, one can also show that

$\sum_{m=1}^{L}(m-1) \psi(m, L)=\frac{8}{(L+1)^{2}} \sum_{k \text { even }} \sum_{k^{\prime} \text { odd }} \frac{\sin ^{2} k \alpha\left(1+\cos k^{\prime} \alpha\right)}{\left(\cos k^{\prime} \alpha-\cos k \alpha\right)\left(2-\cos k \alpha-\cos k^{\prime} \alpha\right)}$

where the range of the sums over $k$ and $k^{\prime}$ are

$$
2 \leqslant k \leqslant L \quad 1 \leqslant k^{\prime} \leqslant L
$$

and

$$
\alpha=\frac{\pi}{L+1} .
$$

The derivation of these two expressions is explained in the appendix.

Remark 2. Except for these two expressions, the general expression for $\psi(m, L)$ is not known. In particular, simple observations, such as the fact that the numerator of $\psi(L, L)$ is always 1, are not yet understood. As we shall see in section 4, knowledge of all the $\psi(m, L)$ would lead to an exact expression for the exponent $\theta(q)$ for all $q$.

From the knowledge of the steady-state properties of the reaction-diffusion problem, one can predict some properties of the spin system. Namely, one can show that for the zero-temperature dynamics of the Potts chain, the probablity $\rho(q, L)$ that starting, with a random initial configuration, a given spin never flips (from $t=0$ until $t=\infty$ ) is

$$
\rho(q, L)=\sum_{m=1}^{L} \psi(m, L) \frac{1}{q^{m-1}} .
$$


Table 2. The probabilities $\psi(m, L)$ of finding $m$ particles on a lattice of $L$ sites in the steady state of the reaction-diffusion model.

\begin{tabular}{|c|c|c|c|}
\hline & & \multirow{7}{*}{$\begin{array}{r}111 \\
1 \\
2 \\
3 \\
1 \\
5 \\
6 \\
\text { stum }\end{array}$} & \multirow{3}{*}{$\begin{array}{r}2 \cdot(1+1 .(j) \\
9112 \\
3865 \cdot 1\end{array}$} \\
\hline & \multirow[b]{2}{*}{$t(m, 2)$} & & \\
\hline III & & & \\
\hline \multirow{3}{*}{$\begin{array}{r}1 \\
2 \\
\text { stum } \\
\end{array}$} & 1 & & 30 \\
\hline & \multirow{2}{*}{$\begin{array}{l}1 \\
2\end{array}$} & & \multirow{2}{*}{$\begin{array}{r}1135 \\
81\end{array}$} \\
\hline & & & \\
\hline iII & $2(m, 3)$ & & $\frac{1}{0976}$ \\
\hline 1 & 2 & (1) & (2) \\
\hline 2 & \multirow{2}{*}{1} & \multirow{2}{*}{1} & \multirow{2}{*}{25086} \\
\hline :3 & & & \\
\hline NHIII & $i$ & $\begin{array}{l}2 \\
3\end{array}$ & 119732 \\
\hline III & $\langle(m, 1)$ & $\ddot{1}$ & 58176 \\
\hline \multirow{2}{*}{$\begin{array}{l}1 \\
2\end{array}$} & s' & 5 & 137566 \\
\hline & \multirow{2}{*}{$\begin{array}{l}23 \\
12\end{array}$} & (; & 200 \\
\hline $\begin{array}{l}2 \\
3\end{array}$ & & $i$ & \multirow{2}{*}{$\begin{array}{r}1 \\
3000101 \\
\end{array}$} \\
\hline $\begin{array}{r}1 \\
\text { sIIII }\end{array}$ & \multirow{2}{*}{$\begin{array}{r}1 \\
41 \\
\end{array}$} & sum & \\
\hline \multirow{2}{*}{$\mathrm{min}$} & & 111 & $3 .(m .8)$ \\
\hline & $4(111.5)$ & 1 & \\
\hline 1 & $(52$ & 2 & (6)69555)36 \\
\hline 2 & 221 & 3 & 3762824 \\
\hline 3 & $17 \mathrm{lli}^{2}$ & 1 & $1746233: 3$ \\
\hline$!$ & 32 & 5 & 773100 \\
\hline 5 & 1 & li & 38718 \\
\hline SHIII & $1 ! 5 \%$ & $\bar{i}$ & 188 \\
\hline & & 8 & 1 \\
\hline & & stlt: & $232 \$ 1608$ \\
\hline
\end{tabular}

\begin{tabular}{|c|c|}
\hline III & $(2 \mathrm{~m}, \mathrm{y})$ \\
\hline$!$ & 119.576380 \\
\hline 2 & $(37(3,5[7.5) \div 2$ \\
\hline 3 & 1112007236 \\
\hline 1 & (6.1.j39.1088 \\
\hline ; & $13.56903 \pi$ \\
\hline (j) & 5838262. \\
\hline$i$ & 218160 \\
\hline 8 & $118 \cdot 1$ \\
\hline 9 & 1 \\
\hline slutil & $26(5) \div 2+13.569$ \\
\hline III & $8(m .1100)$ \\
\hline 1 & $2 0 4 7 4 \longdiv { 3 7 5 9 2 }$ \\
\hline 2 & $12: 3496972192$ \\
\hline 3 & $22339937 \pi 1328$ \\
\hline 4 & 149099841000 \\
\hline 5 & $3856006447: 37$ \\
\hline (;) & $37(15492761$ \\
\hline 7 & $122922 \cdot 178$ \\
\hline 8 & 1221342 \\
\hline y & 2865 \\
\hline 10 & I \\
\hline stum & 560355000496 \\
\hline III & $\psi^{\prime \prime}(m, 11)$ \\
\hline 1 & 638868.1874754 \\
\hline 2 & 40708419309844 \\
\hline 3 & 80209940784610 \\
\hline 4 & 60481733418930 \\
\hline 5 & 18319389302802 \\
\hline 6 & 2202785988876 \\
\hline $\bar{i}$ & $9151755 \times 822$ \\
\hline 8 & {$[5] 28 \times 27648$} \\
\hline 9 & 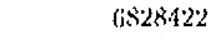 \\
\hline 10 & 6924 \\
\hline 11 & l \\
\hline 111 & $208 \cdot 11200355016334$ \\
\hline
\end{tabular}

\begin{tabular}{|c|c|}
\hline III & $l(m, ! 2)$ \\
\hline I & 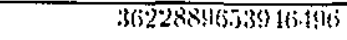 \\
\hline 2 & 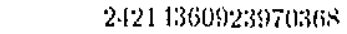 \\
\hline 3 & 512.21197.30117.2352 \\
\hline 4 & 42707.17180 .523201080 \\
\hline i) & 118370979118233311 \\
\hline 6 & $21 \cdot 18168313 \leq 15223$ \\
\hline $\bar{i}$ & $12111001969) 230$ \\
\hline 8 & $265986711+8491$ \\
\hline 9 & $16(0.3 .394) 14.36$ \\
\hline 10 & 382015653 \\
\hline [1] & $|\operatorname{li\pi } 2|$ \\
\hline 12 & \\
\hline sim & $13 \times 85 \div 163333572261824$ \\
\hline$m$ & $2 \cdot(\mathrm{m} . \mathrm{I}: 3)$ \\
\hline I & 3725652928411.1581886 \\
\hline 2 & $25073557.1563856016+96360$ \\
\hline 3 & 5844555288131013898.1 \\
\hline 4 & 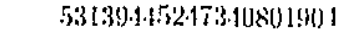 \\
\hline is & $20750631 \cdot 1+440790915 \pi \times 0$ \\
\hline 6 & 3502168958728.5178 .56 \\
\hline $\bar{\imath}$ & $24 \times 61998653013718116$ \\
\hline 8 & $701576175146+7360$ \\
\hline 9 & $72121835757 \% 80$ \\
\hline 10 & 238763.471 .424 \\
\hline 11 & $21.12(15200$ \\
\hline l2 & $40: 384$ \\
\hline 13 & 1 \\
\hline sim & 165792717008810701287 \\
\hline ii) & $4(3 m .11)$ \\
\hline ! & 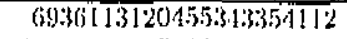 \\
\hline 2 & $502014933570529322 \times 575104$ \\
\hline 3 & $119310287.13854794350(32272$ \\
\hline 4 & 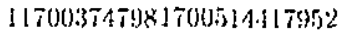 \\
\hline 5 & $50555756(632655655 \times 6(6257)$ \\
\hline b) & 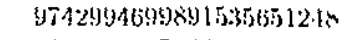 \\
\hline $\bar{\imath}$ & $822037265003354273+1463$ \\
\hline 8 & 290554748594702695999 \\
\hline 9) & $101461517508733325 !$ \\
\hline 10 & $1068771+10.1255 \times 27$ \\
\hline 11 & 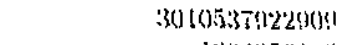 \\
\hline 12 & $12013,2(5180$ \\
\hline [i3 & $(9 \div 50.5$ \\
\hline 1.1 & 1 \\
\hline נII! & 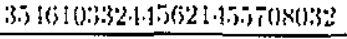 \\
\hline
\end{tabular}

This expression can be understood by using the same argument which was used in section 2 to derive expression (2): the equivalence between the spin model and the reaction-diffusion model. Assume that one starts the dynamics of the reaction-diffusion model at $t=0$ and call $P(m, t)$ the probability that there are $m$ particles in the system at time $t$. For a finite system of $L$ sites, $P(m, t) \rightarrow \psi(m, L)$ as $t \rightarrow \infty$. Therefore in the infinite time limit, (2) becomes (10).

Remark 3. For an infinite system, the probability $r(q, t)$ that a given spin has never flipped 
up to time $t$ decreases to zero as $t \rightarrow \infty$. This is not the case for a finite system: for a finite system there is a finite probability $\left(q^{1-L}\right)$ that initially all spins are in the same state and obviously $\rho(q, L) \geqslant q^{1-L}$.

Using the results given in table 2 for $\psi(m, L)$, one finds for the probability $\rho(q, L)$ that, for a random initial condition, a spin never flips between $t=0$ and $t=\infty$,

$$
\begin{aligned}
& \rho(q, 1)=1 \\
& \rho(q, 2)=\frac{q+1}{2 q} \\
& \rho(q, 3)=\frac{2 q^{2}+4 q+1}{7 q^{2}} \\
& \rho(q, 4)=\frac{8 q^{3}+23 q^{2}+12 q+1}{44 q^{3}} \\
& \rho(q, 5)=\frac{62 q^{4}+224 q^{3}+176 q^{2}+32 q+1}{495 q^{4}} \\
& \rho(q, 6)=\frac{912 q^{5}+3864 q^{4}+3983 q^{3}+1135 q^{2}+81 q+1}{9976 q^{5}}
\end{aligned}
$$

and so on.

Clearly, for $q=1$, one has $\rho(1, L)=1$ for all $L$ : the 'random' initial configuration is the configuration where all spins are the same. Thus the dynamics is trivial and nothing ever moves.

\section{Finite-size scaling}

Finite-size scaling is a well known method to obtain accurate estimates of critical exponents by calculating exactly the properties of finite systems and by analysing their size dependence $[23-25,20,21]$. Using the analogy with the reaction-diffusion model, the $\rho(q, L)$ are exactly known for arbitrary $q$ up to size $L=14$. (One could certainly do better as the size of the transfer matrix is $2^{L-1}$ and the matrix is sparse.) Let us assume that $\rho(q, L)$ decays algebraically for large $L$ :

$$
\rho(q, L) \sim L^{-\theta^{\prime}(q)} .
$$

This defines an exponent $\theta^{\prime}(q)$ which is, in principle, related to the exponent $\theta(q)$ of (1) by [6]

$$
\theta^{\prime}(q)=2 \theta(q)
$$

This relation can be justified by the fact that there is a characteristic length $l(t)$ growing with $t^{1 / 2}$ and that the dynamics stops when $l(t)$ becomes comparable to the system size as there is a single domain left in the system.

The first estimate of $\theta(q)$ one can make based on (11) and (12) is

$$
\theta(q)=-\frac{1}{2} \frac{\log [\rho(q, L+1) / \rho(q, L)]}{\log [(L+1) / L]} .
$$

The results of these estimates for $q=2$ and $q=\infty$ are given for $1 \leqslant L \leqslant 13$ in column 0 of tables 3 and 4 . The convergence is rather slow but looks regular and one can try to extrapolate the data of column 0 . If one makes a polynomial extrapolation of the data of column 0 by taking $k$ consecutive values $x_{L}, x_{L+1}, \ldots, x_{L+k-1}$ of column zero and by using 
Table 3. Estimates of the exponent $\theta(q)$ for $q=2$. The column 0 is obtained using the finitesize scaling expression (12). Columns $1-6$ are polynomial extrapolations of degree $1-6$ of the data of column 0 .

\begin{tabular}{llllllll}
\hline & \multicolumn{7}{c}{$q=2$} \\
\cline { 2 - 7 }$L$ & 0 & $\mathrm{I}$ & 2 & 3 & 4 & 5 & 6 \\
\hline 1 & 0.207519 & 0.313634 & 0.360881 & 0.371070 & 0.371517 & 0.373260 & 0.374975 \\
2 & 0.260576 & 0.345132 & 0.368523 & 0.371428 & 0.372970 & 0.374730 & 0.375187 \\
3 & 0.288761 & 0.356827 & 0.370266 & 0.372456 & 0.374227 & 0.375073 & 0.375037 \\
4 & 0.305778 & 0.362203 & 0.371361 & 0.373468 & 0.374756 & 0.375049 & 0.374983 \\
5 & 0.317063 & 0.365255 & 0.372264 & 0.374112 & 0.374918 & 0.375009 & 0.374989 \\
6 & 0.325095 & 0.367258 & 0.372957 & 0.374470 & 0.374964 & 0.374998 & 0.375000 \\
7 & 0.331118 & 0.368683 & 0.373461 & 0.374668 & 0.374980 & 0.374999 & 0.375004 \\
8 & 0.335814 & 0.369744 & 0.373823 & 0.374781 & 0.374988 & 0.375001 & \\
9 & 0.339584 & 0.370560 & 0.374084 & 0.374850 & 0.374993 & & \\
10 & 0.342681 & 0.371201 & 0.374276 & 0.374894 & & & \\
11 & 0.345274 & 0.371713 & 0.374418 & & & & \\
12 & 0.347477 & 0.372130 & & & & & \\
13 & 0.349374 & & & & & & \\
\hline
\end{tabular}

Table 4. Estimates of the exponent $\theta(q)$ for $q=\infty$. The column 0 is obtained using the finite-size scaling expression (12). Columns $1-6$ are polynomial extrapolations of degree $1-6$ of the data of column 0 .

\begin{tabular}{llllllll}
\hline & \multicolumn{7}{c}{$q=\infty$} \\
\cline { 2 - 7 }$L$ & 0 & 1 & 2 & 3 & 4 & 5 & 6 \\
\hline 1 & 0.500000 & 0.880182 & 1.024671 & 0.979200 & 0.946464 & 0.988116 & 1.016309 \\
2 & 0.690091 & 0.976508 & 0.990568 & 0.953012 & 0.981174 & 1.012281 & 1.004275 \\
3 & 0.785564 & 0.983538 & 0.968034 & 0.971786 & 1.003393 & 1.006277 & 0.996287 \\
4 & 0.835057 & 0.977337 & 0.969910 & 0.989848 & 1.005196 & 0.999617 & 0.997456 \\
5 & 0.863513 & 0.974861 & 0.978455 & 0.997522 & 1.002096 & 0.998321 & 0.999578 \\
6 & 0.882071 & 0.975888 & 0.985605 & 0.999555 & 1.000209 & 0.999007 & 1.000297 \\
7 & 0.895474 & 0.978317 & 0.990255 & 0.999816 & 0.999662 & 0.999652 & 1.000272 \\
8 & 0.905829 & 0.980970 & 0.993123 & 0.999760 & 0.999658 & 0.999938 & \\
9 & 0.914178 & 0.983401 & 0.994933 & 0.999726 & 0.999766 & & \\
10 & 0.921100 & 0.985498 & 0.996132 & 0.999738 & & & \\
11 & 0.926955 & 0.987270 & 0.996964 & & & & \\
12 & 0.931981 & 0.988761 & & & & & \\
13 & 0.936349 & & & & & & \\
\hline
\end{tabular}

the polynomial $P$ of degree $k$ which satisfies $x_{L+i}=P(1 /(L+i))$ for $0 \leqslant i \leqslant k-1$, one obtains the data of column $k$.

We see that for $q=2$ and $q=\infty$, the convergence is greatly improved and leads to accurate estimates of $\theta(q)$. However, one cannot choose polynomials of too high a degree because, as the degree of the extrapolation increases, the value obtained is more and more sensitive to rounding errors. This is probably why one cannot see much improvement for $k>4$.

From the data of tables 3 and 4 , one can estimate that

$$
\theta(2)=0.3750 \pm 0.0001
$$


and

$$
\theta(\infty)=1.0000 \pm 0.0005
$$

For $q=\infty$, one recovers the exact prediction [6] that $\theta(\infty)=1$ and the results of table 4 serve as a test of the accuracy of the extrapolation procedure.

As the $\rho(q, L)$ are known for arbitrary $q$, it is, of course, no problem to repeat these calculations for other choices of $q$. They lead to the estimates given in table 1 for $\theta(q)$.

\section{5. $\epsilon$ expansion and bounds}

One can exploit some of the results which are known exactly on finite systems to calculate the exponent $\theta(q)$ for $q$ close to 1 :

$$
q=1+\epsilon
$$

Equation (10) then becomes

$$
\rho(1+\epsilon, L)=1-\epsilon \sum_{m=1}^{L}(m-1) \psi(m, L)+O\left(\epsilon^{2}\right)
$$

and one can use the exact result (9) of section 3 . The large $L$ dependence of the expression (9) is dominated by small values of $k$ and $k^{\prime}$ and one finds that for large $L$

$$
\sum_{m=1}^{L}(m-1) \psi(m, L) \simeq \frac{4}{\pi} \log L .
$$

Then using (11) and (12), one gets

$$
\theta(q)=(2 / \pi) \epsilon+O\left(\epsilon^{2}\right)
$$

Therefore for small $\epsilon$, one has $\theta(q) \simeq 0.6366 \epsilon$ and this agrees rather well with the estimate for $\epsilon=0.01$ reported in table 1 (the small discrepancy being due to the order $\epsilon^{2}$ ).

Remark 4. There is a situation in which exponents corresponding to non-integer values of $q$ could be measured directly in simulations. Assume that one considers the Ising model with a non-zero average magnetization $\mu$ in the initial condition. Initially each spin is either up with a probability $\frac{1}{2}(1+\mu)$ and down with a probability $\frac{1}{2}(1-\mu)$. Let us call $r_{+}(t)$ the probability that a spin initially up never flips between time 0 and time $t$ and $r_{-}(t)$ the same quantity if the spin is initially down. By the same reasoning as for equation (10), one can show that

$$
r_{ \pm}(t)=\sum_{m=1}^{L} P(m, t)\left(\frac{1 \pm \mu}{2}\right)^{m-1} .
$$

Comparing this with $(10)$ leads to $r_{ \pm}(t)=r\left(q_{ \pm}, t\right)$ with

$$
q_{ \pm}=\frac{2}{1 \pm \mu} \text {. }
$$

Clearly $\mu \rightarrow 1$ corresponds to an initial situation in which almost all spins are identical to the one at the origin, that is $q$ close to 1 , and $\mu \rightarrow-1$ corresponds to $q \rightarrow \infty$ as almost all spins in the system are different from the one at the origin. 
Relation (10) also gives rigorous bounds for the exponent $\theta(q)$. If one chooses two values $q_{1}$ and $q_{2}$ with $q_{1}<q_{2}$, one can write

$$
q_{2} \rho\left(q_{2}, L\right)=\sum_{m=1}^{L} \psi(m, L) q_{2}^{-m}=\sum_{m=1}^{L} \psi(m, L)\left[q_{1}^{-m}\right]^{\log q_{2} / \log q_{1}} .
$$

Then using Jensen's inequality (for a positive random variable $x$, one has $\left\langle x^{\gamma}\right\rangle \geqslant\langle x\rangle^{\gamma}$ when $\gamma \geqslant 1)$, one finds

$$
q_{2} p\left(q_{2}, L\right) \geqslant\left[q_{1} \rho\left(q_{1}, L\right)\right]^{\log q_{2} / \log q_{1}}
$$

This obviously leads to

$$
\theta^{\prime}\left(q_{2}\right) \leqslant \theta^{\prime}\left(q_{1}\right) \frac{\log q_{2}}{\log q_{1}}
$$

and therefore to (4). As $\theta(q)$ is known for $q$ close to 1 , this leads for $q>1$ to

$$
\theta(q) \leqslant \frac{2}{\pi} \log q
$$

For example this bound predicts $\theta(2)<0.44127$ which is clearly satisfied by the estimate $\theta(2) \simeq 0.3750$ of table 1 .

\section{Conclusion}

In this paper, the calculation of the fraction of spins which have never flipped in the zero-temperature dynamics of the one-dimensional Potts model has been related to the properties of a simple reaction-diffusion model. This analogy has been used to obtain accurate estimates for the exponent $\theta(q)$ for any $q$, the first term of an $\epsilon$-expansion for $q$ close to 1 and bounds on this exponent.

The numerical estimate indicates $\theta(q)$ is very close to $3 / 8$ for $q=2$ and it would certainly be of interest to see whether this prediction is exact. More generally, we have seen that some properties (8), (9) of the reaction-diffusion model can be obtained exactly. With more work, one could try to push further the $\epsilon$ expansion or to obtain a large $q$ expansion (this would require an exact expression of $\psi(2, L)$ for all $L$ ). One can even hope to find a complete analytic solution of the reaction-diffusion model. This would automatically lead to the exact expression of $\theta(q)$ for all $q$.

\section{Acknowledgments}

I thank the Isaac Newton Institute for Mathematical Sciences, Cambridge, where this work was started, for its hospitality, and A J Bray, J L Cardy, D Dhar, M Droz, C Godrèche, V Hakim, P L Krapivsky, V Pasquier and G Watts for useful discussions. Special thanks are due to $\mathrm{H}$ Flyvbjerg for his encouragement.

\section{Appendix A.}

In this appendix some of the steady-state properties of the reaction-diffusion model are calculated exactly. These properties give, as special cases, the results (8), (9). We consider the reaction-diffusion model on a ring of $L$ sites with the origin located at position $L$, i.e. the site 0 ( $\equiv$ site $L$ ) is always occupied. Then during any time interval $\mathrm{d} t$, each particle in the system jumps with probability $\mathrm{d} t / 2$ to its left and $\mathrm{d} t / 2$ to its right. When two particles 
occupy the same site, they instantaneously coalesce $A+A \rightarrow A$. Also if the particle at the origin jumps to one of its neighbours, the origin is instantaneously filled by a new particle.

Let us call $f(i, j)$ the probability that in the steady state, all the sites $k$ between $i$ and $j$ are empty $(i \leqslant k \leqslant j$ ). Clearly with this definition, the probability that site $i$ is empty is just $f(i, i)$. Moreover as the origin is always occupied, one has for all $i$ and $j$

$$
f(0, j)=f(i, L)=0 \text {. }
$$

During each time interval $\mathrm{d} t$, it is possible to write a steady-state equation for $i<j$ :

$$
\begin{aligned}
\frac{\mathrm{d} t}{2}[f(i+1, j)-f(i, j)]+\frac{\mathrm{d} t}{2}[f(i, j-1)-f(i, j)] \\
\quad=\frac{\mathrm{d} t}{2}[f(i, j)-f(i-1, j)]+\frac{\mathrm{d} t}{2}[f(i, j)-f(i, j+1)] .
\end{aligned}
$$

The left-hand side of this equation is the probablility of entering into a configuration where all sites between $i$ and $j$ are empty. This happens either if all sites between $i+1$ and $j$ are empty with a particle present at site $i$ (first term) or if all sites between $i$ and $j-1$ are empty with a particle present at site $j$ (second term). The right-hand side can be understood as the probability of leaving the configuration. The case $i=j$ has to be treated separately, and one finds that (A2) is replaced by

$d t[1-f(i, i)]=\frac{\mathrm{d} t}{2}[f(i, i)-f(i-1, i)]+\frac{\mathrm{d} t}{2}[f(i, i)-f(i, i+1)]$.

Clearly (A2) and (A3) are equivalent to

$$
4 f(i, j)=f(i-1, j)+f(i+1, j)+f(i, j-1)+f(i, j+1)
$$

and

$$
4 f(i, i)=2+f(i-1, i)+f(i, i+1) .
$$

The solution for $f(i, j)$ is then

$$
f(i, j)=\sum_{k \text { even }} \sum_{k^{\prime} \text { odd }} \frac{8 \sin k \alpha \sin k^{\prime} \alpha\left[\sin k i \alpha \sin k^{\prime}(j+1) \alpha-\sin k^{\prime} i \alpha \sin k(j+1) \alpha\right]}{(L+1)^{2}\left(\cos k^{\prime} \alpha-\cos k \alpha\right)\left(2-\cos k \alpha-\cos k^{\prime} \alpha\right)}
$$

where

$$
\alpha=\frac{\pi}{L+1} \quad 2 \leqslant k \leqslant L \quad 1 \leqslant k^{\prime} \leqslant L .
$$

Then (8) and (9) follow as

$$
\psi(1, L)=f(1, L-1)
$$

and

$$
L-1-\sum_{i=1}^{L-1} f(i, i)=\sum_{m=1}^{L}(m-1) \psi(m, L)
$$

(this last expression just means that the average occupation of site $i$ is just $1-f(i, i)$ ).

The easiest way to check that (A6) is the solution of (A4)-(A5) is numerically on a computer. It is also possible to derive a proof based on the following trigonometric identities:

$$
\begin{aligned}
\frac{\sin x \sin y[\sin i x \sin (j+1) y-\sin i y \sin (j+1) x]}{\cos y-\cos x} \\
=\sum_{p=0}^{j-i} \sum_{q=0}^{j+i} \sin (p+q-j) x \sin x \sin (q-p-i) y \sin y
\end{aligned}
$$


and for $-L \leqslant n \leqslant L$

$$
\begin{aligned}
& \sum_{k \text { even }} \sin k \alpha \sin n k \alpha=\frac{L+1}{4}\left[\delta_{n,-L}-\delta_{n,-1}+\delta_{n, l}-\delta_{n, L}\right] \\
& \sum_{k^{\prime} \text { odd }} \sin k^{\prime} \alpha \sin n k^{\prime} \alpha=\frac{L+1}{4}\left[-\delta_{n,-L}-\delta_{n,-1}+\delta_{n, 1}+\delta_{n, L}\right]
\end{aligned}
$$

where $k, k^{\prime}$ and $\alpha$ satisfy (A7).

\section{References}

[I] BrayA J 1990 J. Phys. A: Math. Gen. 23 L67

[2] Rácz Z 1985 Phys. Rev. Lett. 551707

[3] Privman V 1992 J. Stat. Phys. 69629

[4] Amar I G and Family F 1990 Phys. Rev. A 413258

[5] Derrida B, Godrèche C and Yekutieli I 1991 Phys. Rev. A 446241

[6] Derrida B, Bray A J and Godrèche C 1994 J. Phys. A: Math. Gen. 27 L357

[7] Stauffer D 1994 J. Phys. A: Math. Gen. 275029

[8] Krapiysky P L, ben-Naim E and Redner S 1994 Phys. Rev. E 502474

[9] Cardy J L 1994 Preprint Oxford

[10] Liggett T M 1985 Interacting Particle Systems (Berlin: Springer)

[11] Spouge J L 1988 Phys. Rev, Lett. 60871

[12] Cheng Z, Redner S and Leyvraz F 1989 Phys. Rev. Lett. 622321

[13] Krapiysky P L 1993 Physica 198A 157

[14] Kang K and Redner S 1984 Phys. Rev. Lett. 52 955; 1985 Phys. Rev. A 32435

[15] Doering C R and ben-Avraham D 1988 Phys. Rev. A 38 3035; 1989 Phys. Rev. Lett. 622563

[16] ben-Avraham D, Burschka M A and Doering C R $1990 \mathrm{~J}$. Stat. Phys. 60695

[17] Leyvraz F and Redner S 1991 Phys. Rev. Lett. 66 2168; 1992 Phys. Rev. A 463132

[18] Cornell S, Droz M and Chopard B 1992 Physica 188A 322

[19] Alcaraz F C, Droz M, Henkel M and Rittenberg V 1994 Ann. Phys. 230250

[20] Krebs K. Pfannmüller M P. Simon H and Wehefritz B 1994 Preprint

[21] Krebs K, Pfannmüller M P, Wehefritz B and Hinrichsen H 1994 Preprint

[22] Newman T J 1994 Preprint

[23] Nightingale M P 1976 Physica 83A 561

[24] Cardy J L 1988 Finite Size Scaling (Amsterdam: North-Holland)

[25] Privman V 1990 Finite Size Scaling and Numerical Simulation of Statistical Mechanics (Singapore: World Scientific) 